\title{
RISK-ADJUSTED GENERAL SURGICAL AUDIT IN OCTOGENARIANS
}

\author{
Kirstin G. Nichols,* David R. Prytherch, $\dagger$ Micheal F. Fancourt,* William T. C. Gilkison,* \\ STEPHEN M. KYLE* AND DAMIEN A. MOSQUERA* \\ *Department of General Surgery, Taranaki Base Hospital, New Plymouth, Taranaki, New Zealand, and $\dagger$ Portsmouth \\ Hospitals NHS Trust, Centre for Healthcare Modelling and Informatics, University of Portsmouth, Portsmouth, UK
}

\begin{abstract}
Background: Surgical admissions in patients more than the age of 80 years are increasing. Age-related comorbidities place this group at particular risk of complications and death. The aim of this study was to specifically document our current outcomes in patients more than 80 years old admitted to a surgical unit, in particular, to assess the risk-adjusted scoring tool used to predict outcomes in this patient population for operative and non-operative patients.

Methods: A prospective audit of all patients older than 80 years admitted to the general surgical unit between the 1 January and 30 November 2006 was carried out. Morbidity and mortality data were collected on standardized pro forma.

Results: There were 243 consecutive admissions in 223 surgical patients (readmission $8.2 \%, n=20$ ) comprising 70 emergency admissions (28.8\%), 82 elective admissions (33.8\%) and 91 non-operative admissions (37.5\%). Complications occurred in $47.1 \%$ of emergency admissions, $18.3 \%$ of elective admissions and $23.3 \%$ of non-operative admissions. Thirty-day mortality was $15.7 \%$ $(n=11)$ for emergency admissions, $0 \%$ for elective admissions and $17.4 \%(n=16)$ for non-operative admissions. Emergency laparotomy 30-day mortality was $31.6 \%(n=6)$. There was no evidence of lack of fit when using the risk-adjusted scoring tool to compare observed with predicted deaths in all patient groups.

Conclusion: In all patients more than the age of 80 years admitted to General Surgery, Taranaki Base Hospital, morbidity and mortality results were acceptable when compared with published work. Risk-adjusted prediction of mortality compared favourably with observed outcomes, but more data are required to validate this tool in elective patients.
\end{abstract}

Key words: aged 80 and over, audit, logistic model, mortality, surgery.

Abbreviations: BHOM, Biochemistry and Haematology Outcome Model; BUPA, British United Provident Association; ICU, intensive care unit; IQ, interquartile; P-POSSUM, Portsmouth Physiological and Operative Severity Score for the enUmeration of Mortality and Morbidity; SRS, surgical risk score.

\section{INTRODUCTION}

Life expectancy has increased significantly in the second half of this century with the ages of the male and female sexes in 2051 expected to be 84 and 88 years, respectively. Currently, the elderly comprise $12 \%$ of New Zealand's population; predictions state that this will increase to $25 \%$ in $2030 . .^{1}$ There are few studies that have investigated this significant, older proportion of our surgical workload and the outcomes associated with carrying out surgery on these patients.

Surgical audit is an annual requirement for continuing certification by the Board of Continuing Professional Development and Standards. ${ }^{2}$ It is important that reported mortality figures in this high-risk group of patients accurately reflect surgical performance. Crude, unadjusted mortality rates do not adjust for different casemixes in the surgical population and are not good indicators of surgical performance.

K. G. Nichols BAS (Physiotherapy), BM, BS; D. R. Prytherch PhD, Csi; M. F. Fancourt FRACS, FRCS (Ed); W. T. C. Gilkison MB, ChB (NZ) FRACS; S. M. Kyle FRACS; D. A. Mosquera MD, FRACS.

Correspondence: Dr Kirstin G. Nichols, Waikato District Health Board, Hamilton 3200, New Zealand.

Email: kirstin_grace@hotmail.com

Accepted for publication 25 April 2008.
The Physiological and Operative Severity Score for the enUmeration of Mortality and Morbidity (POSSUM), ${ }^{3-5}$ PortsmouthPOSSUM (P-POSSUM) ${ }^{6,7}$ and Surgical Risk Score (SRS) can be used to predict 30-day mortality after adjusting for casemix. ${ }^{8}$ POSSUM requires collection of 12 physiological variables and 6 operative variables, which is difficult and time-consuming to implement into regular audit practice, ${ }^{9}$ with only $30 \%$ being fully completed in one study. ${ }^{9}$ POSSUM, P-POSSUM and SRS are models that require operative data and are not suitable for non-operative patients 10 although they constitute a substantial part of the surgical workload. 9 POSSUM has also been shown to overpredict mortality in low-risk patients and underestimate mortality in elderly and emergency patients. The SRS discriminates well for low-risk procedures, but requires further validation for high-risk procedures. ${ }^{11}$

A model will be more useful if it applies to all surgical admissions, both operative and non-operative, as well as high-risk and low-risk procedures. Most surgeons accept that the mode of presentation, physiological condition of the patient and surgical procedure carried out, are predictors of patient outcome.11,12 The Biochemistry and Haematology Outcome Model (BHOM) has been proposed by Prytherch et al. 2003, which includes age, sex, type of admission, physiological parameters and operative severity score. ${ }^{10}$ Predicted mortalities are calculated using a derived logistic regression equation. The BHOM uses information, which is easily obtainable from most hospital information systems. 
The aim of this study was to document our current outcomes in patients older than 80 years admitted to a surgical unit. In particular, to assess the risk-adjusted scoring tool used to predict outcomes in operative and non-operative patients developed by Prytherch et al.

\section{METHODS}

A prospective audit was conducted of all consecutive octogenarian General Surgical admissions from 1 January to 30 November 2006 excluding day case admissions. All four general surgeons participated in the audit.

Pro forma - agreed by all surgeons contained the minimum clinical dataset - haemoglobin, white cell count, sodium, potassium, urea, age on admission, sex, British United Provident Association (BUPA) operative severity score (completed on discharge for operative admissions), mode of admission and 30-day mortality as described by Prytherch et al.10,13 BUPA operative severity score is widely used in the private medical sector in the UK. This has been shown to be a useful measure of surgical workload and complexity of operation. ${ }^{14}$

The main end-points for this study included the complications for each patient, 30-day mortality, discharge destinations and calculation of the BHOM for operative and non-operative patients.

Data were collected by the general surgical house officers. Each week the pro forma was collated, cross-referenced against daily in-patient lists and discharge database. The data were entered into an Excel database by a single registrar.

The Excel database was used to collate data and calculate predicted mortality using the logistic regression equation. There are three separate models: elective admission, emergency admission and emergency admission that did not undergo an operation referred to as non-operative admission.

The logistic regression equation for predicting mortality is as follows:

$$
\begin{aligned}
\operatorname{L\eta }\left\{\frac{R}{1-R}\right\}= & \text { constant } \\
& +(\beta 1 \times \text { sex })+(\beta 2 \times \text { age on admission }) \\
& +(\beta 3 \times \text { urea })+(\beta 4 \times \text { sodium }) \\
& +(\beta 5 \times \text { potassium })+(\beta 6 \times \text { haemoglobin }) \\
& +(\beta 7 \times \text { white cell count }) \\
& +\beta \text { operative severity score }
\end{aligned}
$$

where $R$ is the risk of death, $\beta$ is the coefficient value given in Table 1 corresponding to patient type and $\beta$ operative severity score is the appropriate coefficient for the operative severity (if applicable) from Table 1. The constants and coefficients are given in Table 1.

\section{Predicted mortality}

The logistic regression equation is applied to the minimum clinical dataset. Using the predicted risk of death, the episodes were grouped into risk ranges chosen from the non-operative patients to give (as close as possible) similar predicted deaths in each range then carried
Table 1. Constants and coefficients for risk models

\begin{tabular}{lcrr}
\hline & $\begin{array}{c}\text { Non- } \\
\text { operative }\end{array}$ & $\begin{array}{c}\text { Emergency } \\
\text { operation }\end{array}$ & $\begin{array}{c}\text { Elective } \\
\text { operation }\end{array}$ \\
\hline Constant & -6.9247 & -5.1412 & -15.4194 \\
Sex $(\mathrm{M}=1),(\mathrm{F}=0)$ & 0.2203 & -0.1734 & 0.3290 \\
Age & 0.0765 & 0.0496 & 0.1145 \\
Urea & 0.0971 & 0.0782 & 0.1110 \\
Sodium & -0.0133 & -0.0067 & -0.0047 \\
Potassium & -0.0487 & -0.2744 & 0.2846 \\
Haemoglobin & -0.0897 & -0.0730 & -0.0383 \\
White cell count & 0.0584 & 0.0424 & 0.0048 \\
BUPA operative severity & score & & \\
1 & - & 0 & 0 \\
2 & - & 0.1485 & -0.2611 \\
3 & - & 0.9012 & 1.0052 \\
4 & - & 1.6405 & 2.0910 \\
5 & - & 1.6501 & 3.3117 \\
6 & - & 1.5592 & 3.1713 \\
7 & - & 3.0636 & 4.4598 \\
8 & - & -2.8362 & 0 \\
\hline
\end{tabular}

BUPA, British United Provident Association; -, no coefficient (value 0).

these over to emergency and elective admissions. The number of episodes within each risk band is given together with the mean risk $(\%)$. The predicted number of deaths is then calculated by the number of episodes multiplied by the mean risk (\%). This is compared with the actual number of deaths. Goodness of fit is assessed using the $\chi^{2}$-test with 4 degrees of freedom (d.f.). This is a null hypothesis test - models with $P$-values greater than 0.05 are considered to show no evidence of lack of fit. Cochrane's rules for $\chi^{2}$ require at least five predicted events in $80 \%$ of risk ranges of strata.

\section{RESULTS}

\section{Patient characteristics}

Overall there were 243 admission episodes from 223 surgical patients with 20 readmissions $(8.2 \%)$ constituting $13 \%$ of all surgical admissions. The male : female ratio was $2: 3$. The median age of patients was 84 (interquartile (IQ) range 82-87 years). There were 70 emergency admissions $(28.8 \%)$, 82 elective admissions $(33.8 \%)$ and 91 non-operative admissions $(37.5 \%)$. There was one elective admission that did not undergo an operation and is included in the non-operative patient group. For BHOM analysis, this patient is excluded, as only acute non-operative admissions can be included in this modelling.

The overall length of stay (LOS) (median and IQ range) was 4 days (3-8 days). Emergency admissions stayed 7.5 days (412 days), which was longer than elective admissions (LOS 3 days, IQ range, 2-7 days) and non-operative admission (LOS 4 days, IQ range $3-5.5$ days).

The overall classification of admissions included colorectal $37.6 \%(n=89)$, vascular $17.6 \%(n=43)$, hepatobilary $9.1 \%$ $(n=22)$, upper gastrointestinal $8.2 \%(n=20)$, cellulitis $6.2 \%$ $(n=15)$, trauma $4.9 \%(n=12)$, hernia $4.9 \%(n=12)$, skin $4.9 \%(n=12)$, general $3.7 \%(n=9)$, head and neck $2.1 \%$ $(n=5)$, breast $1.2 \%(n=3)$ and thoracic $0.05 \%(n=1)$. The level of complexity according to BUPA operative severity score for elective admissions were 15 minor, 31 intermediate, 15 major, 9 major+, 2 CMO1, 2 CMO2, 8 CMO3 and emergency admissions they were 25 minor, 17 intermediate, 16 major, 2 CMO1 and no $\mathrm{CMO} 2 / 3$ scores. There were no CMO4 scores. 


\section{Complications}

Blood transfusions were required in $20 \%(n=14)$ emergency patients, $8.5 \%(n=7)$ elective patients and $9 \%(n=8)$ nonoperative patients. Complications outlined in Table 2 occurred in 33 emergency patients $(47.1 \%), 15$ elective patients $(18.3 \%)$ and 22 non-operative patients $(23.3 \%)$. Five patients required return to theatre $(3.3 \%)$, four emergency patients and one elective patient. Thirty-day mortality rates were $15.7 \%(n=11)$ for emergency admission and $17.4 \%(n=16)$ for non-operative admissions. There were no elective deaths. There were 19 emergency laparotomies carried out with 6 deaths $(31.6 \%)$.

Intensive care unit (ICU) admissions: there were six emergency patients in ICU with a total stay of 24 days and five elective patients with a total stay of 9 days. High-dependency unit admissions: there were 17 emergency patients with a total stay of 57 days, 3 elective patients with a total stay of 9 days and 3 non-operative patients totalling 6 days.

Transfers during admission to another medical team was required for $9 \%(n=6)$ emergency patients, $5 \%(n=4)$ elective patients and $9 \%(n=8)$ non-operative patients. Rehabilitation was necessary for $14 \%(n=10)$ of emergency patients and $10 \%$ $(n=9)$ of non-operative patients with no elective patients needing rehabilitation services. Discharge destinations showed most of patients in all groups returned home or to previous rest-home placement: $79 \%(n=55)$ emergency patients, $98 \%(n=80)$ elective patients and $77 \%(n=70)$ non-operative patients. New resthome referrals were generated for $13 \% \quad(n=9)$ emergency patients, $2 \%(n=2)$ elective patients and $15 \%(n=14)$ nonoperative patients. Hospice and death in emergency patients was $1 \%(n=1)$ and $7 \%(n=5)$, respectively, and in the nonoperative patients $2 \%(n=2)$ and $6 \%(n=5)$, respectively.

\section{Predicted mortality}

The logistic regression equation was applied to the minimum clinical dataset. Risk stratification is shown in Tables 3-5. Emer-

Table 2. Complications

\begin{tabular}{lccc}
\hline & $\begin{array}{c}\text { Emergency, } \\
n=33\end{array}$ & $\begin{array}{c}\text { Elective, } \\
n=15\end{array}$ & $\begin{array}{c}\text { Non- } \\
\text { operative, } \\
n=22\end{array}$ \\
\hline Cardiac & 17 & 8 & 5 \\
Respiratory & 14 & 3 & 7 \\
Renal & 13 & 10 & 15 \\
Thrombosis/bleeding & 3 & 2 & 1 \\
Wound & 7 & 2 & 0 \\
Systemic/other & 2 & 1 & 3 \\
Total & 55 & 24 & 31 \\
\hline
\end{tabular}

gency, elective and non-operative admission patient groups show no evidence for lack of fit. Cochrane's rules for $\chi^{2}$ require at least five predicted events in $80 \%$ of risk ranges of strata therefore at present there are inadequate numbers to prove in this audit; however, these results are promising.

\section{DISCUSSION}

Elderly patients are higher-risk patients with longer LOS and higher rates of complications. There are limited studies describing complication and mortality rates for this population, especially non-operative patients admitted under surgical care. Our results confirm higher rates of complications particularly in emergency patients.

Mortality rates were also high in the emergency and nonoperative groups at 15.7 and $17.8 \%$, respectively. This is similar to other reports of emergency mortality rates between 11.1 and $29 \% .15-17,19,20$ In addition, emergency laparotomy mortality was found to be $31.6 \%$, which was comparable to a similar study, which reported $60 \%$ mortality. 18 The variability of mortality rates reflects the limited and small studies investigating mortality in this patient population. The high non-operative mortality rate is a figure frequently not reported, as most papers focus on surgical operative deaths. This is a neglected group of patients, which require more attention in future surgical audit.

There were no elective surgical deaths in octogenarians and an $18 \%$ complication rate. These figures compare well with published reports of elective operative mortality rates of $7.5 \%$ and morbidity rates of $33 \% .15-17$

Interestingly, discharge destinations were similar for emergency and non-operative patients with 79 and $77 \%$ returning home or previous rest-home residence. All elective patients returned to their previous residence, be that home or rest home. New rest-home referrals were required for $13 \%$ of emergency patients and $15 \%$ of non-operative patients. Other research has found that $70 \%$ of general surgical patients were discharged home and $16 \%$ discharged to rest home, which correlated to our results. ${ }^{20}$

The BHOM, which was used to analyse our patient data, has been proposed by Prytherch et al.10 as an alternative to POSSUM, ${ }^{3-5}$ P-POSSUM 6,7 and SRS, which can be used to similarly predict 30 -day mortality. ${ }^{8}$ Criticism of these current models is, most notably, the difficulty in collecting the physiological and operative variables required to incorporate these tools into regular clinical practice. 9

The formula for POSSUM and P-POSSUM is complex and performance depends on the method of analysis and also requires manipulation for different subspecialties within surgery. POSSUM has been shown to overpredict mortality in low-risk patients and underestimate in elderly and emergency patients.

Table 3. Minimum clinical dataset applied to emergency admissions

\begin{tabular}{lcccr}
\hline Risk band (\%) & No. episodes & Mean risk $(\%)$ & Predicted deaths & Observed deaths \\
\hline $0-14$ & 46 & 6.86 & 3 & 4 \\
$15-22$ & 8 & 17.93 & 1 & 3 \\
$23-38$ & 11 & 27.14 & 3 & 3 \\
$38-100$ & 5 & 52.09 & 3 & 1 \\
$0-100$ & 70 & 14.54 & 10 & 2.08 \\
\hline
\end{tabular}

$\chi^{2}=4.39,4$ d.f., $P=0.357$. No evidence of lack of fit. 
Table 4. Minimum clinical dataset applied to elective admissions

\begin{tabular}{lcccr}
\hline Risk band $(\%)$ & No. episodes & Mean risk $(\%)$ & Predicted deaths & Observed deaths \\
\hline $0-14$ & 68 & 2.52 & 2 & 0 \\
$15-22$ & 4 & 17.51 & 1 & 0 \\
$23-38$ & 6 & 25.41 & 2 & 0 \\
$38-100$ & 4 & 49.18 & 2 & 0 \\
$0-100$ & 82 & 7.2 & 6 & 0.85 \\
\hline
\end{tabular}

$\chi 2=8.52,4$ d.f., $P=0.074$. No evidence of lack of fit.

Table 5. Minimum clinical dataset applied to non-operative admissions

\begin{tabular}{lcccr}
\hline Risk band (\%) & No. episodes & Mean risk $(\%)$ & Predicted deaths & Observed deaths \\
\hline $0-14$ & 48 & 8.9 & 4 & 2 \\
$15-22$ & 20 & 17.63 & 4 & 5 \\
$23-38$ & 13 & 30.45 & 4 & 3 \\
$38-100$ & 9 & 50.28 & 5 & 6 \\
$0-100$ & 90 & 18.09 & 16 & 0.75 \\
\hline
\end{tabular}

$\chi^{2}=3.37,4$ d.f., $P=0.498$. No evidence of lack of fit.

Surgical Risk Scores in comparison to POSSUM is based on the Confidental Enquiry into PeriOperative Deaths grade, American Society of Anesthesiologists grade and the BUPA operative grade 8 and performs well in low-risk procedures. SRS performs well in lowrisk procedures, as the lowest predicted risk is $0.07 \%$. The absolute minimum risk of mortality for POSSUM is $1.08 \%$ and $0.2 \%$ for P-POSSUM, which is too high for these procedures. ${ }^{8}$ An imoportant limitation is that only operative patients can be assessed, as none of these tools is suitable for non-operative patients.

Overall, a risk assessment scoring system should have few variables, be easy to collect and implemented into daily practice, be available for every patient and have limited potential observer bias. BHOM uses clinical data, which is easily available from a single venesection. It is logically feasible to collect this for all patients as part of routine care. The data are objective and cannot be manipulated by observer bias.

The BHOM risk-adjusted tool for predicting mortality in emergency, elective and non-operative admission patient groups shows no evidence for lack of fit when applied to our audit data. Unfortunately, Cochrane's rule for $\chi^{2}$ require at least five predicted events in at least $80 \%$ of strata therefore at present there are inadequate numbers to prove in this audit; however, these results are promising. The main difficulty would be in proving elective operations, as we had no patient deaths in this category. Prytherch et al. in the original paper, found similar problems with insufficient numbers, which prevented stratification into more than five bands. Large patient numbers would be required, which is not easily obtainable in a small peripheral hospital. The BHOM appears, albeit with insufficient numbers, appropriate for our population and adequately predicted mortality. Further studies will be required to ascertain if this is the case for Australasian population, preferably in a larger centre to gain the numbers required.

\section{CONCLUSION}

In conclusion, this general surgical audit confirms the outcomes in the more-than-eighties were acceptable when compared with other studies. The minimum clinical dataset is a promising tool for risk-adjusted evaluation of surgical mortality, which can be applied easily in a provincial setting and deserves wider attention.

\section{REFERENCES}

1. Dunstan K, Thomson N. Demographic Aspects of New Zealand's Aging Population. Statistics New Zealand, Ministry of Social Development. [Cited Mar 2006.] Available from URL: www. stats.govt.nz/products-and-services/papers/demographic-aspectsnz-ageing-population.html

2. Watters DAK, Green AJ, Van Rij A. Guidelines for surgical audit in Australia and New Zealand. ANZ J. Surg. 2006; 76: 78-83.

3. Neary WD, Heather BP, Earnshaw JJ. The physiological and operative severity score for the enumeration of mortality and morbidity (POSSUM). Br. J. Surg. 2003; 90: 157-165.

4. Copeland GP, Jones D, Walters M. POSSUM: a scoring system for surgical audit. Br. J. Surg. 1991; 78: 355-60.

5. Whitely MS, Prytherch DR, Higgins B, Weaver PC, Prout WG. An evaluation of the POSSUM surgical scoring system. Br. J. Surg. 1996; 83: 812-15.

6. Wijesinghe LD, Mahood T, Scott DJA, Berridge DC, Kent PJ, Kester RC. Comparison of POSSUM and Portsmouth predictor equation for predicting death following vascular surgery. $\mathrm{Br} . \mathrm{J}$. Surg. 1998; 85: 209-12.

7. Prytherch DR, Whitely MS, Higgins B, Weaver PC, Prout WG, Powell SJ. POSSUM and Portsmouth POSSUM for predicting mortality. Br. J. Surg. 1998; 85: 1217-20.

8. Sutton R, Bann S, Brooks M, Sarin S. The surgical risk scale as an improved tool for risk-adjusted analysis in comparative surgical audit. Br. J. Surg. 2002; 89: 763-8.

9. Bann SD, Sarin S. Comparative audit: the trouble with POSSUM. J. R. Soc. Med. 2001; 94: 632-4.

10. Prytherch DR, Sirl JS, Weaver PC, Schmidt P, Higgins B, Sutton GL. Towards a national clinical minimum data set for general surgery. Br. J. Surg. 2003; 90: 1300-305. 
11. Brooks MJ, Sutton R, Sarin S. Comparison of surgical risk score, POSSUM and p-POSSUM in higher risk surgical patients. $B r . J$. Surg. 2005; 92: 1288-92.

12. Hartley MN, Sagar PM. The Surgeon's 'gut feeling' as a predictor of post-operative outcome. Ann. R. Coll. Surg. Engl. 1994; 76 (Suppl): 277-8.

13. Prytherch DR, Briggs JS, Weaver PC, Schmidt P, Smith GB. Measuring clinical performance using routinely collected data. Med. Inform. Internet Med. 2005; 30: 151-6.

14. Jones SM, Collins CD. Caseload or workload? Scoring complexity of operative procedures as a means of analysing workload. $\mathrm{Br}$. Med. J. 1990; 301: 324-5.

15. Walsh TH, Audit outcome of major surgery in the elderly. $B r . J$. Surg. 1996; 83: 92-7.
16. Isbister WH. Colorectal Surgery in the elderly: an audit of surgery in Octogenarians. Aust. N. Z. J. Surg. 1997; 67: 557-61.

17. Abbas S, Booth M. Major abdominal surgery in octogenarians. NZ Med. J. 2003; 116: 1172. Available from URL: www.nzma.org.nz/journal/116-1172/402/

18. Krysa J, Sandison A. Emergency admissions of surgical patients aged 80 and over: audit of outcome. Ann. R. Coll. Surg. Engl. 2005; 88: 323-4.

19. Gollop SJ, Fancourt MW, Gilkison WTC, Kyle SM, Mosquera DA. Prospective audit of colorectal resections in a peripheral public hospital. ANZ J. Surg. 2006; 76: 817-20.

20. Rørbæk-Madsen M, Dupont G, Kristensen K, Holm T, Sørensen J, Dahger H. General surgery in patients aged 80 years and older. Br. J. Surg. 1992; 79: 1216-18. 